\title{
Perception's Crucial Role in Radiology Education
}

\author{
Richard B. Gunderman MD, PhD
}

Parth Patel BS

Indiana University School of Medicine, 702 North Barnhill Drive, Room 1053, Indianapolis, IN 46202

\section{Key Words}

Perception; education; radiology; radiologists

Perception is at the core of what radiologists do every day. Almost by definition, fully qualified radiologists are very good at perceiving, at least when it comes to the detection and interpretation of radiological images of the human body. But could a more thorough understanding of perception — and in particular, how we learn to perceive - enable radiologists to find more joy in their work, further enhance their powers of perception, or teach radiology more effectively?

\section{PERCEPTION}

The word perception comes from the Latin percipere, meaning to comprehend or grasp with the mind. It in turn is derived from two roots, per- meaning thoroughly and kap- meaning to grasp. So to perceive something is to grasp it thoroughly and thereby to see it for what it is. When novices viewing a chest radiograph mistake a skin fold for a pneumothorax, they have in a sense failed to perceive or misperceived what they are seeing.

Implicit in this example is the notion that we learn to perceive. We are born with the ability to see, but it is only over time and often with effort that we develop the ability to see what is really before us.

Automobile enthusiasts learn to distinguish between different makes and models of cars, fashionistas gain

This is the author's manuscript of the article published in final edited form as:

Gunderman, R. B., \& Patel, P. (2019). Perception's Crucial Role in Radiology Education. Academic Radiology, 26(1), 141-143. https://doi.org/10.1016/j.acra.2018.08.004 
an appreciation for different styles of dress, and birdwatchers learn to tell the difference between different avian species.

Likewise, students of radiology learn to distinguish between normal and abnormal anatomic patterns, as well as between different types of pathologic processes. On what basis, for example, is it possible for someone inspecting a skull radiograph to distinguish between a cranial suture and a nondisplaced skull fracture? Likewise, how might a learner begin to distinguish between different types of skull lesions, such as infectious and neoplastic processes?

\section{LEARNING TO PERCEIVE}

Such questions remind us that there are several different forms of perceptual learning (1). One is distinguishing between different objects of perception, such as dogs and rabbits. Many features enter into such a distinction, such as the fact that dogs have their eyes mounted on the fronts of their heads, while rabbits' are placed more on the sides. In radiology, one such distinction might involve osteolytic versus osteoblastic lesions.

Another type of perceptual learning involves recognizing the similarities or connections between different things. For example, though different from each other in many respects, dogs and rabbits are both animals. In radiology, learners acquire the ability to recognize anatomic boundaries, and to distinguish imaging artifacts, such as a fold in a blanket, from a pathologic lesion, such as pneumoperitoneum, based on whether such findings respect anatomic boundaries.

A third form of perceptual learning concerns the ability to direct attention where attention is due. In sports such as tennis, for example, novices may devote unnecessary attention to their opponent's movements, while experts have developed the ability to keep their eye on the ball (2). Art experts tend to focus on structural features of artwork whereas naïve art viewers tend to focus on objects in artwork (3). Likewise, expert radiologists know what key features of the brain, lungs, and liver they most need to attend to to detect and identify key pathologies. 


\section{HOW PERCEPTUAL LEARNING WORKS}

For some time, researchers supposed that enhancements in perceptual abilities reflected cognitive changes. To put this in crude neuroanatomic terms, it was assumed that the visual portions of the brain were unchanged, and that all the learning was taking place in the higher associative regions. However, over the last several decades, it has become apparent that many such changes are taking place in the sensory parts of the brain themselves. Studies have shown that damage to tracts connecting the visual cortex with the prefrontal cortex leads to problems in object recognition (4). The right fusiform gyrus and right occipital lobe have been implicated in automotive expertise (5).

Such findings support the concept of perceptual learning, as distinct from other domains of learning, such as mathematics. When a novice and an expert radiologist both look at a chest radiograph, they are to some extent literally seeing different things. For one thing, the expert's eye is drawn to certain parts of the radiograph more than others, and the expert recognizes connections and distinctions that the novice cannot perceive (6).

For example, the novice does not know if the heart is enlarged, if the lungs are edematous, or if pleural effusions are present. The expert, however, not only perceives each of these features almost instantly, but simultaneously combines them into a provisional impression such as congestive heart failure. As novices progress along the path to expertise, they are not merely accumulating facts but refashioning their perceptual systems.

Here it is important to distinguish between perceptual development and perceptual learning. In normal infants, a variety of perceptual capacities develop, such as the ability to recognize faces and to determine which direction sounds are coming from. Such abilities appear to be largely innate. Other abilities, however, such as distinguishing between interstitial and airspace pulmonary edema, are not part of normal human development and are acquired only through specialized education. In other words, visual expertise is not an automatic perceptual skill and requires attention and semantic skill (7). 


\section{EXPERTISE}

Simply put, experts have learned to perceive things that nonexperts have not. But this is only part of the story. Not only do experts see things that escape the notice of nonexperts, they also know what to attend to and what to ignore. In many sports, for example, expert defenders learn to attend less to where a ballhandler's head is directed than to their hips, precisely because in doing so they are less likely to be "faked out" (8).

The best experts take this principle even further. All-time great ice hockey star Wayne Gretzky famously said that he skated not to where the puck was located, but the where the puck was going to be. In other words, experts can not only select out the most salient features of a static image, but also integrate what they see at any particular instant into an event or process unfolding over time, in a way somewhat analogous to a medical prognosis.

When novices perform a procedure such as a biopsy or abscess drainage, they tend to follow a generic set of instructions. When experts do the same, they adapt in real time to what they see. For example, suppose the patient has anomalous anatomy. Because experts see the procedure not as a prescribed series of steps but a goal that can be reached by a variety of paths, they are able to adapt their performance to best meet the demands of the situation at hand.

The perception of experts is strategic. They are less interested in following any particular set of instructions than in achieving their goal in as safe, effective, and even elegant a manner as possible. They perceive each aspect of a case - the patient, the equipment, other personnel, and a host of other factorsin the larger context of the mission at hand. To an important degree, experts are defined in part by the way they see what they are doing.

In chess, after experts view a board position for just a split second, they can correctly reproduce the position of each of the pieces of the board. However, they can only do so if the position they are shown corresponds to an actual game, and not merely a random arrangement of pieces. Experts break the 
chessboard down into so-called chunks, arrangements of pieces that have meaning in the context of the game (9). Chess skill is correlated with the ability to remember and manipulate larger chunks (10). Expertise in perception is only possible when what we see makes strategic sense.

\section{IMPLICATIONS FOR RADIOLOGY EDUCATION}

This perspective on perceptual learning has important implications for education. For one thing, if we want the education of medical students, residents, fellows, and practicing radiologists to be oriented toward an ultimate goal of expertise, then educators need to emphasize wherever possible the strategic dimension of understanding. Learners need to be focused not only on what to know and do, but also on the why. Only with a firm grasp of the why is it possible to adapt like an expert to the situation at hand.

Of course, an understanding of perceptual learning also reveals pitfalls in radiology education. For example, learners may learn to draw incorrect distinctions, such as "I don't need to know this, because it will never be on the test." Or they may lump things together that should be kept separate—-"This was on last year's test, so I need to know it for this year." Or they may put undue emphasis on material— "I know this is clinically irrelevant, but they keep putting this sort of thing on the test." In each of these cases, they will suffer to some degree from miseducation.

The same applies to a hot new area of interest in radiology, machine learning. Some proponents suppose that if machines can correctly identify imaging features as well or better than humans, then machines should supplement or replace humans. Yet machines have no conscience, and left to their own devices, they can develop sexist and racist tendencies (11). The machine neither knows what it is doing nor what it should do, and as a result, it operates in moral blindness.

For this reason, as we educate radiologists' eyes and brains, we need to ensure that the education of their hearts proceeds apace. No matter how good the eyes of radiologists, if they come to regard patients as anatomic specimens and fail to recognize their underlying humanity, then the development of their 
refined perceptual capabilities will have been for naught. Perception is shaped not only by theories but also by the character of those doing the perceiving.

\section{References}

1. Connolly K. Perceptual learning: the flexibility of the senses. New York, NY: Oxford University Press, 2019.

2. Del Villar F, Garcia Gonzalez L, Iglesias D, et al. Expert-novice differences in cognitive and execution skills during tennis competition. Percept Mot Skills 2007; 104:355-365.

3. Vogt S, Magnussen S. Hemispheric specialization and recognition memory for abstract and realistic pictures: a comparison of painters and lay-men. Brain Cogn 2005; 58:324-333.

4. Gilaie-Dotan S, Harel A, Bentin S, et al. Neuroanatomical correlates of visual car expertise. Neuroimage 2012; 62:147-153.

5. Gauthier I, Skudlarski P, Gore JC, et al. Expertise for cars and birds recruits brain areas involved in face recognition. Nat Neurosci 2000; 3:191-197.

6. Bertram R, Kaakinen J, Bensch F, et al. Eye movements of radiologists reflect expertise in CT study interpretation: a potential tool to measure resident development. Radiology 2016; 281:805-815.

7. Harel A. What is special about expertise? Visual expertise reveals the interactive nature of realworld object recognition. Neuropsychologia 2016; 83:88-99.

8. Fujii K, Shinya M, Yamashita D, et al. Anticipation by basketball defenders: an explanation based on the three-dimensional inverted pendulum model. Eur J Sport Sci 2014; 14:538-546.

9. Gobet F, Simon HA. Expert chess memory: revisiting the chunking hypothesis. Memory 1998; 6:225-255. 
10. Gong Y, Ericsson KA, Moxley JH. Recall of briefly presented chess positions and its relation to chess skill. PLoS One 2015; 10:e118756.

11. Garg N, Schiebinger L, Jurafsky D, et al. Word embeddings quantify 100 years of gender and ethnic stereotypes. Proc Natl Acad Sci U S A 2018; 115:E3635-E3644. 